\title{
ON THE CONJUGACY OF INJECTORS
}

\author{
GRAHAM A. CHAMBERS
}

ABSTRACT. In their paper, Injektoren endlicher aufiösbarer Gruppen, Fischer, Gasehütz and Hartley ask the following question. If $\mathfrak{F}$ is a normal subgroup closed class of groups and if $G$ is a finite solvable group which possesses $\mathfrak{F}$-injectors, is it true that any two $\mathfrak{F}$ injectors of $G$ are conjugate in $G$ ? A partial answer is given. It is proven that if $G$ has $p$-length 1 for each prime $p$, then the answer to this question is yes.

1. Introduction. Fitting classes and injectors were introduced by Fischer, Gaschütz and Hartley [2]. A Fitting class $\mathfrak{F}$ is an isomorphism closed class of groups satisfying $f_{1}: G \in \mathfrak{F}, N \triangleleft G$ implies $N \in \mathfrak{F}$, $f_{2}: N_{1}, N_{2} \triangleleft G, N_{1}, N_{2} \in \mathfrak{F}$ implies $N_{1} N_{2} \in \mathfrak{F}$. If $G$ is a group, $V \leqq G$ is an $\mathfrak{F}$-injector of $G$ provided $N \triangleleft \triangleleft G$ implies $V \cap N$ is $\mathfrak{F}$-maximal in $N$. Satz 1 [2] states that if $\mathfrak{F}$ is a Fitting class and $G$ is a finite solvable group, then $G$ possesses $\mathfrak{F}$-injectors and any two are conjugate. At the close of [2] the authors ask if the conjugacy of injectors can be proven using only the first of the defining properties of a Fitting class. That is, if $\mathfrak{F}$ is an isomorphism closed class of groups satisfying $f_{1}$ and if $G$ is a finite solvable group which possesses $\mathfrak{F}$-injectors, is it true that any two $\mathfrak{F}$-injectors of $G$ are conjugate? A partial answer is given. We prove that if $G$ has $p$-length 1 for each prime $p$, then the answer to this question is yes.

2. p-normally embedded subgroups. In proving our result we will use the concept of a $p$-normally embedded subgroup. $V \leqq G$ is said to be $p$-normally embedded in $G$ if a Sylow $p$-subgroup $V_{p}$ of $V$ is also Sylow in some normal subgroup of $G$. This concept was introduced by Hartley [3] and has also been studied in [1]. We are going to need the following theorem which is essentially a restatement of Theorem 2.6 of $[1]$.

Theorem 1. Let $G$ be a finite solvable group and suppose $V \leqq G$ is $p$ normally embedded in $G$ for each prime $p$. Suppose $W \leqq G$ and that for each prime $p$ the Sylow p-subgroups of $W$ are conjugate to those of $V$. Then $V$ and $W$ are conjugate.

We are also going to need the following theorem which will be used

Received by the editors June 10, 1970.

AMS 1969 subject classifications. Primary 2025, 2040.

Key words and phrases. Finite solvable group. Fitting class, injector, $p$-normally embedded subgroup. 
to show that if $G$ has $p$-length 1 for each prime $p$, then the $\mathfrak{F}$-injectors of $G$ are $p$-normally embedded in $G$.

Theorem 2. Let $p$ be a prime and let $G$ be a p-solvable finite group. Then $G$ has p-length 1 if and only if each p-subgroup of $G$ is Sylow in some subnormal subgroup of $G$.

Proof. Suppose $G$ has $p$-length 1 and that $P$ is a $p$-subgroup of $G$. Let $K=O_{p^{\prime}}(G)$ and consider $G / K . P K / K$ is a $p$-subgroup of $G / K$ and, if $K \neq 1, P K / K$ is Sylow in some $L / K \triangleleft \triangleleft G / K$ by induction. But then $P$ is Sylow in $L \triangleleft \triangleleft G$ as required. Thus we may assume $K=1$. Then $G$ has a normal Sylow $p$-subgroup $P^{*}$ and $P \triangleleft \triangleleft P^{*} \triangleleft G$ so that $P \triangleleft \triangleleft G$.

To prove the converse we suppose each $p$-subgroup of $G$ is Sylow in some subnormal subgroup of $G$. If $N \triangleleft G$ and $P / N$ is a $p$-subgroup of $G / N$, then there is a $p$-subgroup $P^{*}$ of $G$ such that $P=P^{*} N$. By assumption $P^{*}$ is Sylow in some $L \triangleleft \triangleleft G$ so that $P / N=P^{*} N / N$ is Sylow in $L N / N \triangleleft \triangleleft G / N$. Thus by induction $G / N$ has $p$-length 1 for any $1 \neq N \triangleleft G$. If $O_{p^{\prime}}(G) \neq 1$, then we are done. Otherwise we can assume $G$ has a unique minimal normal subgroup $K$ which is a $p$ group. If $\Phi(G) \neq 1$, then $G / \Phi(G)$ has $p$-length 1 and hence so does $G$. Thus we may assume $\Phi(G)=1$ so that $K$ is complemented. Assume $M K=G$ and $M \cap K=1$. If $M$ is $p^{\prime}$, then $K$ is Sylow $p$ in $G$ and we are done. Suppose then that $1 \neq M_{p}$ is Sylow $p$ in $M$. By assumption $M_{p}$ is also Sylow in some $L \triangleleft \triangleleft G$. Since $K$ is a $p$-group and $M_{p} \cap K=1, L$ is a proper subgroup of $G$. But then there exists a proper normal subgroup $L^{*}$ of $G$ such that $M_{p} \leqq L \leqq L^{*}$. Since $K$ is the unique minimal normal subgroup of $G, K \leqq L^{*}$. Then $M_{p} K \leqq L^{*}$ so that $L^{*}$ has $p^{\prime}$ index. Now each $p$-subgroup of $L^{*}$ is Sylow in some $R \triangleleft \triangleleft G$ and so is Sylow in $L^{*} \cap R \triangleleft \triangleleft L^{*}$. Thus $L^{*}$ has $p$-length 1 by induction. Since $L^{*}$ has $p^{\prime}$ index this implies $G$ has $p$-length 1 . Q.E.D.

\section{The main theorem.}

TheOREM 3. Suppose $G$ has p-length 1 for each prime p and suppose $V$ and $W$ are $\mathfrak{F}$-injectors of $G$ where $\mathfrak{F}$ is an isomorphism closed class of groups satisfying $f_{1}$. Then

(1) $V$ is p-normally embedded in $G$ for each prime $p$.

(2) V and $W$ are conjugate.

Proof. The proof is by induction on $|G|$. We assume both statements have been shown to hold whenever $|G|<n$. Now assume $|G|=n$. Our first step is to show that $|V|=|W|$. Let $M$ be a maximal normal subgroup of $G$. $V \cap M$ and $W \cap M$ are each $\mathfrak{F}$-injectors of 
$M$ and so they are conjugate by induction. Suppose in fact that $V^{0} \cap M=(V \cap M)^{o}=W \cap M$. If $W=W \cap M$, then $W \leqq V^{o}$ and, since $W$ and $V^{o}$ are each $\varsubsetneqq$-injectors, this would imply $W=V^{o}$. Certainly $|W|=|V|$ in this case. Thus we may assume $W M=G$ and $|W|$ $=[G: M]|W \cap M|$. Similarly we may assume $|V|=[G: M]|V \cap M|$ and once again we have $|W|=|V|$.

Let $V_{p}$ and $W_{p}$ denote Sylow $p$-subgroups of $V$ and $W$ respectively. Our second step is to show that $V_{p}$ and $W_{p}$ are conjugate. If both $V_{p}$ and $W_{p}$ are Sylow in $G$, this is clear. Suppose then that $V_{p}$ is not Sylow in $G$. From Theorem 2 we know $V_{p}$ is Sylow in some proper subnormal subgroup $L$ of $G$. $V \cap L$ and $W \cap L$ are each $₹$-injectors of $L$ and so they are conjugate by induction. Choose $g$ such that $V \cap L$ $=(W \cap L)^{o} \leqq W^{0}$. Then $V_{p}$ is Sylow in $V \cap L \leqq W^{0}$ so that $V_{p}$ is contained in some conjugate of $W_{p}$. Since $V$ and $W$ have the same order so do $V_{p}$ and $W_{p}$ and so we conclude that $V_{p}$ and $W_{p}$ are conjugate.

The next step is to show that $V$ is $p$-normally embedded in $G$. By Theorem 2, $V_{p}$ is Sylow in some $L \triangleleft \triangleleft G$. If $L=G$, then $V_{p}$ is Sylow in $G$ so that $V$ is $p$-normally embedded in $G$. If $L$ is proper then there is a proper normal subgroup $H$ of $G$ such that $V_{p} \leqq L \leqq H . V \cap H$ is an $\mathfrak{F}$-injector of $H$ and $V_{p}$ is Sylow in $V \cap H$. Since $H<G, V \cap H$ is $p$ normally embedded in $H$ by induction. That is, $V_{p}$ is Sylow in some normal subgroup $K$ of $H$. But then $V_{p}$ is Sylow in $\left(V_{p}\right)^{H} \leqq K$. Suppose now that $\alpha \in \operatorname{Aut}(H)$. Then $(V \cap H)^{\alpha}$ is again an $\mathfrak{F}$-injector of $H$ and since $|H|<|G|,(V \cap H)^{\alpha}$ is conjugate to $V \cap H$ in $H$ by induction. In particular $\left(V_{p}\right)^{\alpha}$ is conjugate to $V_{p}$ in $H$. This shows that $\left(V_{p}\right)^{H}$ is in fact characteristic in $H \triangleleft G$. But then $V_{p}$ is Sylow in $\left(V_{p}\right)^{H} \triangleleft G$ so that $V$ is $p$-normally embedded in $G$ as required.

As a final step we invoke Theorem 1 to complete the proof that $V$ and $W$ are conjugate. Q.E.D.

\section{REFERENCES}

1. G. A. Chambers, p-normally embedded subgroups of finite soluble groups, J. Algebra 16 (1970), 442-455.

2. B. Fischer, W. Gaschütz and B. Hartley, Injektoren endlicher auflösbarer Gruppen, Math. Z. 102 (1967), 337-339. MR 36 \#6504.

3. B. Hartley, On Fischer's dualization of formation theory, Proc. London Math. Soc. (3) 19 (1969), 193-207. MR 39 \#5696.

University of Alberta, Edmonton, Alberta, Canada 